\section{Gewalt in der Psychiatrie}

Drohungen, Beleidigungen und manchmal auch körperliche Übergriffe - nicht nur in der Forensik müssen Psychiater und Therapeuten mit aggressiven Patienten rechnen. Darauf deutet eine aktuelle Umfrage der DGPPN unter ihren Mitgliedern. Insgesamt hatten 308 Ärzte und Psychologen daran teilgenommen.

Praktisch alle klinisch tätigen und $88 \%$ der ambulant arbeitenden Teilnehmer hatten angegeben, schon einmal in ihrem $\mathrm{Ar}$ beitsleben in der Psychiatrie verbal oder körperlich angegriffen worden zu sein, erläuterte Dr. Dirk Richter von der Berner Fachhochschule. Bei zwei Drittel der stationär und der Hälfte der ambulant tätigen Befragten geschahen solche Übergriffe in den zwölf Monaten vor der Umfrage. Der Großteil davon ließ sich jedoch auf verbale Attacken zurückführen. Immerhin 37\% der in Kliniken arbeitenden Befragten (Niedergelassene 13\%) waren aber auch schon einmal körperlich von Patienten angegriffen worden. Am häufigsten sind Übergriffe bei medizinischen Tätigkeiten ohne Körperkontakt sowie bei der Aufnahmeprozedur.

Besonders gefährdet für Gewalthandlungen sind offenbar junges Pflegepersonal, Auszubildende und unerfahrene Mitarbeiter. Darauf deutet eine Untersuchung von über 330 körperlichen Übergriffen in der forensischen Psychiatrie, die Dr. Nahlah Saimeh vorstellte. Mit großem Abstand am häufigsten waren Nein-Botschaften die Auslöser - den Patienten wurde ein Wunsch verwehrt. Auf dem zweiten Platz folgten Streitigkeiten mit anderen Patienten.

Als präventive Maßnahmen nannte Saimeh eine gute therapeutische Beziehung zu den Patienten, Mitarbeiter, die in Deeskalation und der Früherkennung von kritischen Situationen gut geschult sind, aber auch ein Deeskalationstraining für Patienten. Als Frühwarnzeichen sieht Saimeh etwa den sozialen Rückzug der Patienten, einen gestörten Schlaf-WachRhythmus, Gereiztheit, Anspannung und verbale Aggression. Zum Schutz der Mitarbeiter müsse es in psychiatrischen Kliniken eine absolute Nulltoleranz gegen Gewalt geben. Ferner seien organisatorische Maßnahmen wie Notfallpläne, Fehlermanagement und standardisierte Abläufe in Krisenfällen essenziell.

(TM)

Symposium „Aggression und Stalking gegenüber Psychiatern und anderen Berufsgruppen in der Psychiatrie"; DGPPN-Kongress, Berlin, 25.-28.11.2015

\title{
Schützen Kakerlaken vor Allergien?
}

Kakerlaken rufen, wo immer sie auftauchen, Ekel und Abscheu hervor; häufige Begegnungen mit den schnellen schwarzen Krabbeltierchen scheinen jedoch aus immunologischer Sicht Vorteile zu haben.

$F^{\text {orden }}$ orscher aus den USA konnten nachweisen, dass eine per Pricktest festgestellte Sensibilisierung gegenüber den Schädlingen bei Hausstaubmilbenallergikern mit einer geringeren Ausprägung allergischer Symptome verbunden ist [He W et al. J All Clin Immunol 2015; 136: 658-66]. Die Studienergebnisse unterstützen die Hygiene-Hypothese, nach der Schmutz und die frühe Auseinandersetzung des Immunsystems mit den darin enthaltenen Mikroorganismen eher vor Allergien schützen als ein sauberes Umfeld. Die Wissenschaftler hatten Allergiker in einer Expositionskammer gezielt an vier aufeinanderfolgenden Tagen über jeweils drei Stunden bestimmten Aeroallergenen ausgesetzt. Die Teilnehmer litten unter Hausstaubmilben- $(\mathrm{M}+)$ und zum Teil auch unter Pollenallergie $(\mathrm{P}+), 43 \%$ waren zusätzlich gegen Kakerlaken sensibilisiert (C+).

Während der Exposition mit Hausstaubmilbenallergen zeigten die $21 \mathrm{M+-}$ Teilnehmer erwartungsgemäß Symptome einer Rhinokonjunktivitis; diese waren am stärksten, wenn zusätzlich eine Pollenallergie, aber keine Sensibilisierung gegen

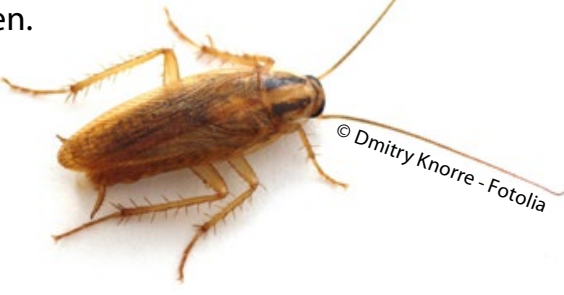

Kakerlaken vorlag, und am schwächsten in der Konstellation M+ P- C+.

Auch eine künstliche Exposition mit Pollen (Zeder, Eiche, Ambrosia) überstanden die C+-Patienten besser. Insgesamt ergab sich eine um den Faktor 2,8 erhöhte Wahrscheinlichkeit, deutlich abgeschwächte Allergiesymptome zu entwickeln. Die Labordiagnostik bestätigte dies: So fanden sich bei „Kakerlakenallergie" erheblich mehr aktivierte CD8+T-Zellen im peripheren Blut.

Kakerlaken sind unbestritten Schädlinge; über Lebensmittel können sie Krankheiten übertragen. Daher sollte man nicht ernsthaft dazu raten, den Kontakt mit ihnen zu suchen. In der Studie war die C+-Sensibilisierung wohl auch nur ein Marker für das Aufwachsen in einem unsauberen Umfeld. (eo)

hautnah dermatologie 2015/6

\section{Myostatinantikörper nützt Älteren nach Sturz}

$\mathrm{N}^{2}$ ach einem Sturz führte eine 24-wöchige Therapie mit einem Myostatinantikörper bei älteren Patienten zu einer Zunahme der fettfreien Körpermasse und zu einer Verbesserung in funktionellen Tests. $\mathrm{Zu}$ diesem Ergebnis kommt eine placebokontrollierte multizentrische doppelblinde Phase-II-Studie, in die insgesamt 201 ältere, muskelschwache Patienten ( $\geq 75$ Jahre) nach einem Sturz aufgenommen wurden [Becker $C$ et al. Lancet Diabetes Endocrinol 3:948-957]. Die Patienten der Verumgruppe bekamen in vierwöchigem $\mathrm{Ab}$ stand insgesamt sechs s.c.-Injektionen des Myostatinantikörpers LY2495655. Patienten der Placebogruppe erhielten
s.c.-Injektionen einer Kochsalzlösung. Nach 24 Wochen lag die DXA-ermittelte Körpermasse in der Verumgruppe um $430 \mathrm{~g}$ höher als in der Placebogruppe. In etwa der Hälfte der eingesetzten Funktionstests zur muskulären Leistungsfähigkeit fand sich eine signifikante Besserung, so im Treppensteigen, im Stuhlaufstehtest und beim schnellen Gehen. Hauptnebenwirkung war in der Verumtherapiegruppe eine lokale Rötung an der Einstichstelle, die bei zwei Verumpatienten zum Therapieabbruch führte.

Zeitschrift für Gerontologie und Geriatrie $1 / 2016$ 